\title{
Intervenciones
}

\section{no destructivas para el diagnóstico de la salud estructural de dos templos de Aguascalientes, en México}

Non-Destructive Interventions for the Diagnosis of the Structural Condition of Two Temples in Aguascalientes, Mexico

\section{Edith Estefanía Orenday Tapia}

Doctorado en Ciencias de los Ámbitos Antrópicos,

Universidad Autónoma de Aguascalientes (UAA), México

eeorenday@gmail.com

\section{Evangelina Tapia Tovar}

Departamento de Sociología y Antropología,

Universidad Autónoma de Aguascalientes (UAA), México

etapia@correo.uaa.mx

Jesús Pacheco Martínez

Departamento de Construcción y Estructuras,

Universidad Autónoma de Aguascalientes (UAA), México

jesus.pacheco@edu.uaa.mx

\section{Resumen}

Este INFORME presenta los trabajos — algunos en proceso y otros concluidos- del grupo de investigación de Ingeniería Estructural de Edificios Patrimoniales (IEEP) de la Universidad Autónoma de Aguascalientes (UAA). Uno de los criterios para incluirlos fue que estuvieran orientados a diagnosticar la salud estructural de templos católicos del Centro Histórico de Aguascalientes, a partir de métodos no destructivos. El INFORME se enfoca en el análisis del templo de San Antonio de Padua y de la Catedral de Aguascalientes, tanto por su relevancia arquitectónica como por ser culturalmente icónica. Entre sus resultados destaca la importancia de utilizar este tipo de intervención en edificios de valor patrimonial con el fin de preservar no sólo su arquitectura sino también su valor sociocultural y religioso.

Palabras clave

patrimonio religioso; métodos no destructivos; diagnóstico estructural; Aguascalientes; México 


\section{Abstract}

This REPORT presents both the works in progress and those completed by the research group of Structural Engineering of Heritage Buildings (IEEP for its acronym in Spanish) of the Universidad Autónoma de Aguascalientes (UAA). One of the criteria in the decision to incorporate them was their orientation to diagnose the structural condition of Catholic temples in the Historic Center of Aguascalientes using non-destructive methods. The REPORT focuses on the analysis of the temple of San Antonio de Padua and the Cathedral of Aguascalientes, due to its architectural relevance and for being culturally iconic. The results highlight the importance of using this type of intervention in buildings that have heritage value in order to preserve not only their architecture but also their sociocultural and religious value.

\section{Keywords}

Religious heritage; non-destructive methods; structural diagnosis; Aguascalientes, Mexico

\section{Introducción: contexto sociocultural}

os templos católicos son un pilar del patrimonio cultural de Aguascalientes, tanto por su importancia arquitectónica como por su significado cultural; el valor que esas edificaciones tiene para sus habitantes se engrandece al ser un símbolo de religiosidad que les da sentido de pertenencia.

El estado de Aguascalientes se localiza en la parte central de México, en una zona caracterizada por su arraigada religiosidad católica. Es considerado "parte del Ilamado bastión católico más tradicional" (Patiño y Zalpa 2014: 63).

Aunque se reconoce que durante los tres últimos decenios se ha dado una reducción de la población católica en Aguascalientes, según datos censales se observa que más de 90\% de sus pobladores se declara católico (Inegi 2010); la Encuesta de Prácticas y Creencias Religiosas, aplicada en Aguascalientes en 2009, señala que casi $77 \%$ de los habitantes de la entidad acude a los templos católicos por lo menos una vez al mes (Patiño y Zalpa 2014).

La arquitectura que se observa en el Centro Histórico de Aguascalientes y en zonas cercanas a éste (Figura 1) es muestra de la importancia de la religión católica en la constitución de la cultura y en la identidad del aguascalentense.

El patrimonio cultural "es una pertenencia, que tal vez pueda ser objetiva, pero ineludiblemente se trata de una apropiación simbólica [...] está relacionada con bienes valiosos - tangibles o intangibles - que deben ser conservados para seguir perteneciendo al grupo en cuestión" (Martínez 2016: 13).

Órdenes religiosas como las de los dieguinos, franciscanos, juaninos, mercedarios y dominicos llegaron a la ciudad y sentaron las bases culturales que su población aún conserva.

\section{Primeros estudios}

Dada la importancia de los templos católicos en la ciudad, se han efectuado estudios y trabajos para preservar la cultura religiosa (Gutiérrez 2003, 2006, 2007a y 2007b; Imdosoc 2015; Muñoz 1996; Zalpa 2003 y 2014). Desde el Ayuntamiento de Aguascalientes se promueven recorridos turísticos por el centro de la ciudad que incluyen, por su belleza arquitectónica, los templos católicos. De igual manera, en los espacios universitarios dedicados a la investigación sociocultural, se llevan a cabo investigaciones para conocer el sentido social que la religión católica asume entre la población del estado (Gutiérrez 2007a; Padilla 2013 y 2009; Patiño 2013; Patiño y Tapia 2009; Patiño y Zalpa 2014; Tapia 2011; Zalpa 2003 y 2013; Padilla 2013).

Ese interés académico también está presente en la arquitectura y la ingeniería; los trabajos al respecto han permitido evaluar los edificios procurando preservar sus valores físicos, arquitectónicos y culturales, sentando así las bases para la preservación estructural y constructiva de los templos. En el viejo continente, desde hace varias décadas, estudios sobre la preservación de edificios históricos han interesado a muchos investigadores. Entre las más importantes asociaciones encargadas de velar por la conservación del patrimonio cultural a escala internacional están la UNESCO y el International Council on Monuments and Sites (ICOMOS), mientras que en el ámbito nacional el Instituto Nacional de Antropología e Historia (INAH) tiene como objeto investigar, conservar y difundir el patrimonio arqueológico, antropológico, histórico y paleontológico de la nación con el fin de fortalecer la identidad y la memoria de la sociedad que lo posee (INAH 2018), y existe también la Dirección General de Sitios y Monumentos.

Sin embargo, están surgiendo nuevas líneas de especialización, centradas en los edificios patrimoniales desde el punto de vista de la ingeniería (SAHC 2019). Los primeros estudios de este tipo que se realizaron a escala nacional, fueron en 1989, cuando la Catedral Metropolitana mostró signos de daño grave (una grieta de tamaño considerable) ocasionado por los hundimientos de la zona; se requería, entonces, una valoración urgente para determinar la estabilidad del edificio. Se formó un comité de expertos de áreas como geotecnia, estructuras y materiales, del Instituto de Ingeniería de la Universidad Nacional Autónoma de México (II-UNAM) y de la Facultad de Arquitectura (FA) de la misma institución, dirigido por el arquitecto Sergio Saldívar. Dicho comité llegó a la conclusión de que el hundimiento era causado por dos factores: el tipo de suelo deformable en el que se encuentra construido (arcillas lacustres) y por razón de que está situado sobre antiguos templos mexicas, que ocasionaban un hundimiento diferencial y éste, a su 


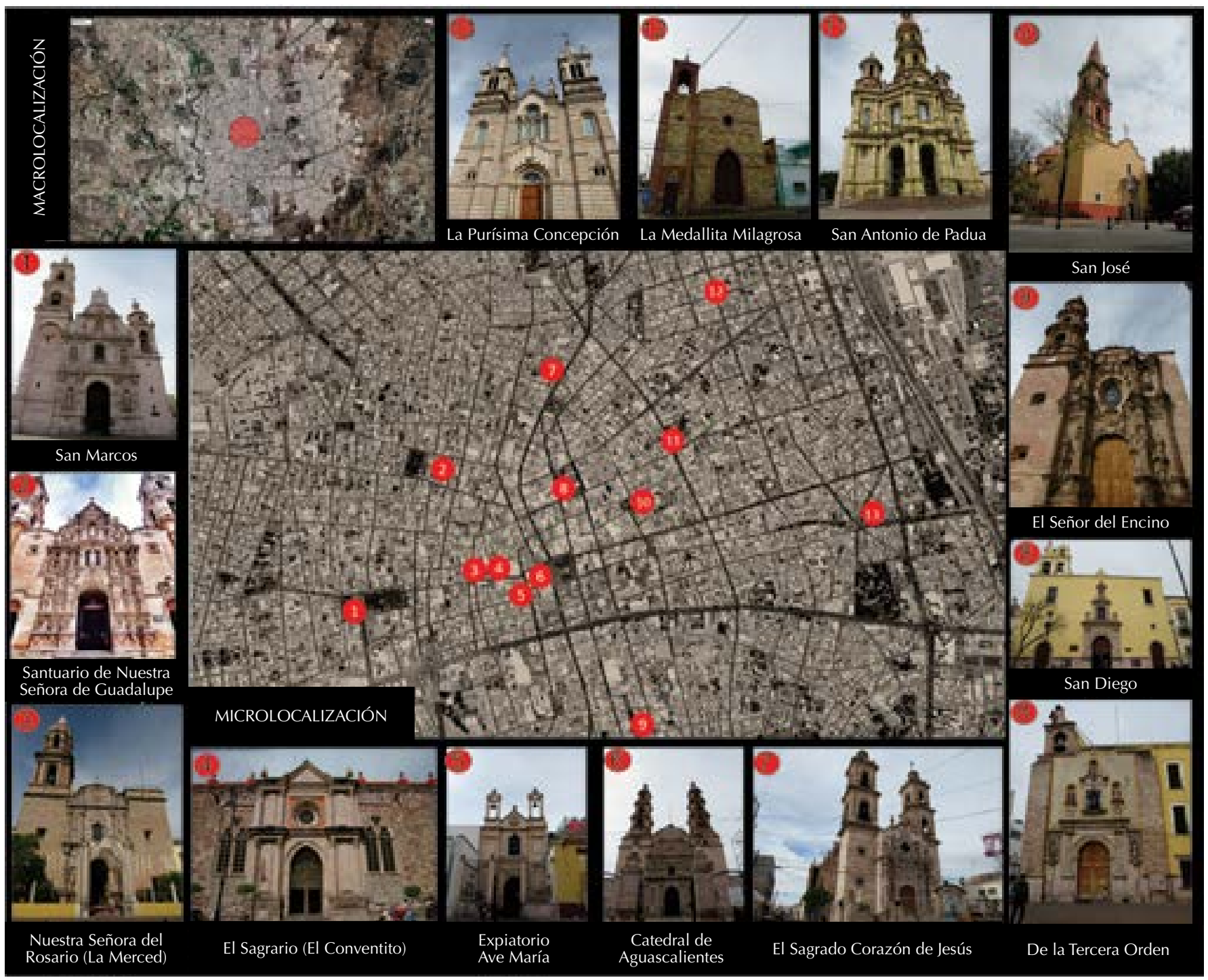

FIGURA 1. Mapa de los templos más antiguos de la ciudad de Aguascalientes (Fotografías: Edith Orenday-Tapia, 2019; mapa: Google Earth ${ }^{\circledR}$, 2018).

vez, un agrietamiento de los elementos estructurales de la catedral (Aguilera Jiménez 2013); esos hallazgos permitieron conocer la importancia que el "efecto sitio" tiene sobre una estructura y la necesidad de ésta de ser evaluada y monitoreada para su preservación.

Recientemente, en la UAA se han comenzado a realizar estudios para el diagnóstico de edificios patrimoniales desde la perspectiva de la ingeniería. Es en ese contexto como este artículo presenta una descripción de las investigaciones emprendidas por la universidad sobre los estudios de recintos religiosos desde la perspectiva de la ingeniería estructural (Ánimas 2015; Ánimas, Navarro, Pacheco, García, Arroyo, Cordero y Esparza 2013; Ánimas, Pacheco y Ortiz 2014; Navarro 2013; Orenday-Tapia, Pacheco-Martínez, Padilla-Ceniceros, Ánimas-Rivera, Ortiz-Lozano y Gaxiola Apodaca 2019; Orenday-Tapia, Pacheco-Martínez, PadillaCeniceros y López-Doncel, 2018; Orenday 2016; Padilla, Pacheco, López y Orenday 2017; Padilla, Sonnemann y Pacheco 2015).
Peña (2008) menciona que una estructura histórica posee dos tipos de valores: los intrínsecos y los físicos. Los valores intrínsecos son aquellos inherentes a la estructura, pero no de manera tangible, como la arquitectura del edificio (estilo arquitectónico, espacio, distribución, uso), el valor artístico (murales, muebles, frescos), el histórico (época de construcción, sucesos acontecidos en ésta), el valor de la ingeniería (técnicas de construcción y materiales usados, que dejan un legado de las capacidades de los constructores de la época) y el valor económico (turismo, atracción).

Este trabajo se propone agregar a los valores intrínsecos de las estructuras el valor sociocultural. En cuanto a los edificios religiosos, éste se refiere a la identidad religiosa, por lo que, en el caso de la ciudad de Aguascalientes, es ese valor adicional que los hidrocálidos le dan a los edificios de culto.

El objetivo principal de una intervención debe ser salvaguardar los valores físicos e intrínsecos de una estructura (Peña 2008), es decir, preservar el edificio sin dañarlo 
físicamente y protegiendo, en la medida de lo posible, todos esos aspectos que le dan valor, siguiendo la teoría de la mínima intervención del edificio como primer criterio de conservación (De Solà-Morales, 2015).

\section{Metodología}

En la evaluación de los dos templos se optó por utilizar técnicas que fueran no destructivas; por ello, se excluyeron todos aquellos estudios que emplearon técnicas destructivas, o que se realizaron en otros edificios religiosos.

Para la elección de los estudios que aquí se presentan, se hizo una investigación documental de los trabajos de diagnóstico y de evaluación hechos por el grupo de investigación de Ingeniería Estructural de Edificios Patrimoniales (IEEP) de la UAA, del que formamos parte, referidos a dos de los edificios religiosos más importantes y bellos de la ciudad: el templo de San Antonio de Padua y la Catedral de Aguascalientes, cuya localización se muestra en la Figura 2.

\section{La Catedral de Aguascalientes}

Desde su construcción fue referencia para la traza de la ciudad, porque forma parte de la plaza de armas de Aguascalientes, junto con el palacio municipal y el palacio de gobierno del Estado (Figura 2A). En una primera etapa
(1621), se construyó la nave central a base de mampostería en bruto. En la Figura 3 se muestran las diferentes etapas de construcción en planta que Padilla (2017) identificó:

La construcción del edificio actual inició en 1704 con el cura Antonio Flores de Acevedo y concluyó en 1738 con el párroco Manuel Colón de Larreategui. Su fachada está conformada por tres cuerpos con un retablo en

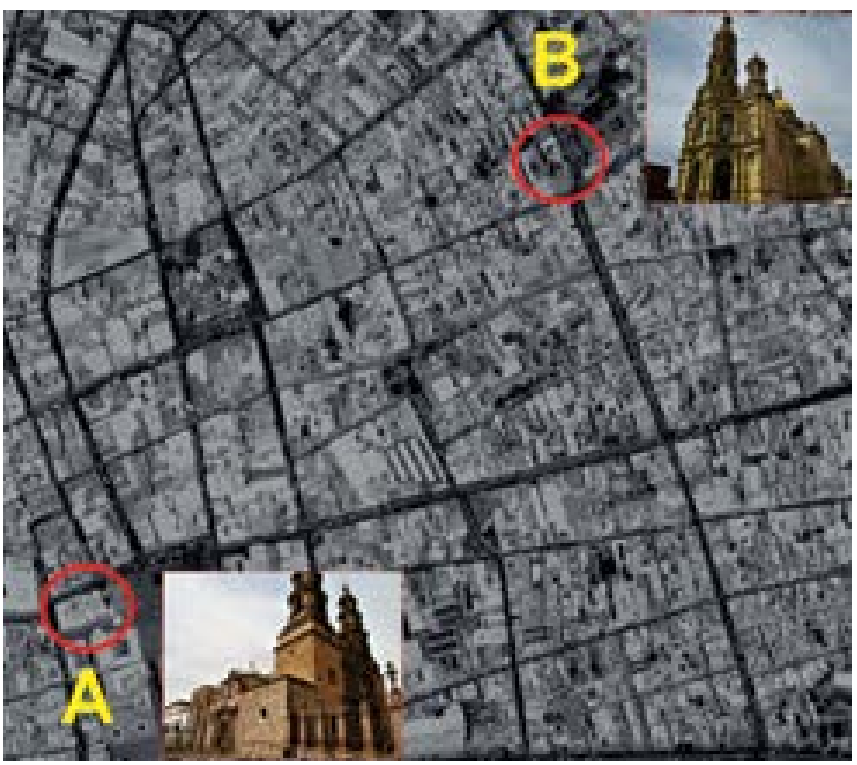

FIGURA 2. Mapa de localización de la Catedral de Aguascalientes y del templo de San Antonio de Padua (Fotografías: Edith Orenday-Tapia, 2019; mapa: Google Earth $\left.{ }^{\circledR}, 2018\right)$.

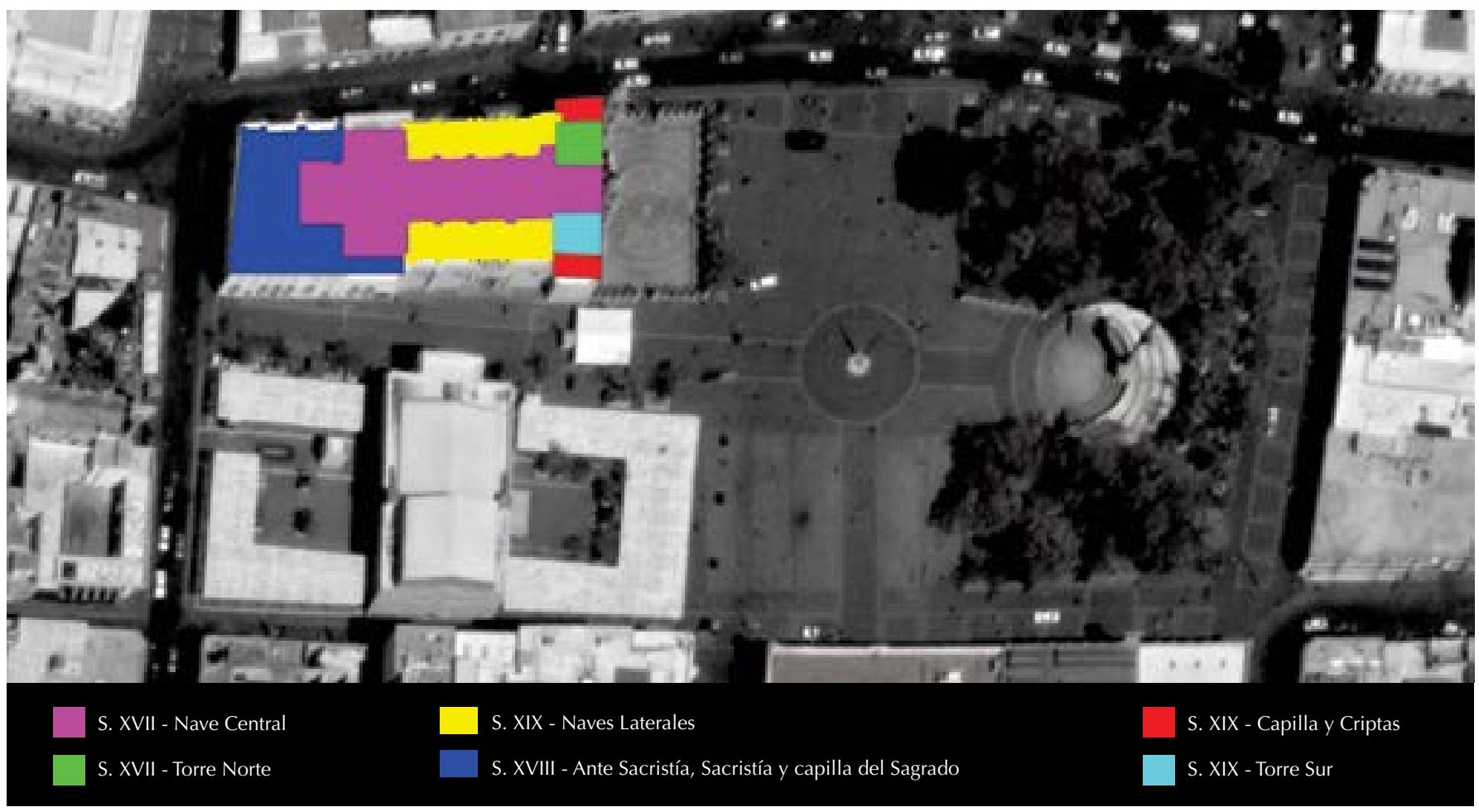

FIGURA 3. Etapas de construcción de la Catedral de Aguascalientes sobre mapa satelital; vista de su entorno (Ilustración: Edith Orenday-Tapia, 2019; fuente: R. Padilla 2017; mapa: Google Earth $\left.^{\circledR}, 2018\right)$. 


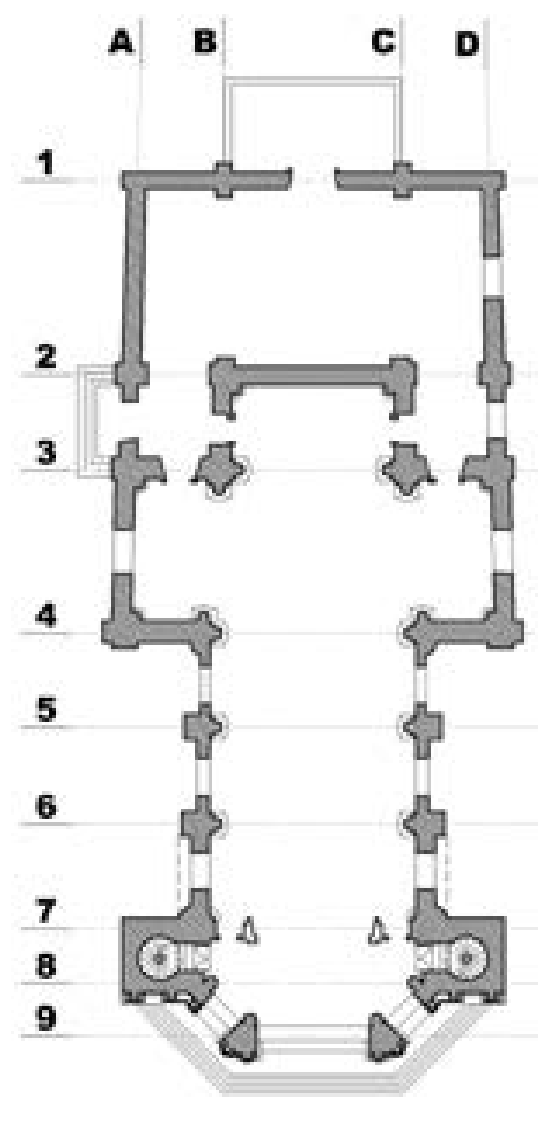

Planta arquitectónica

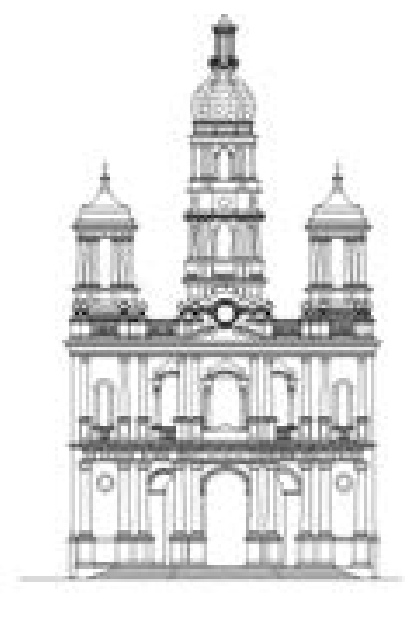

Fachada principal

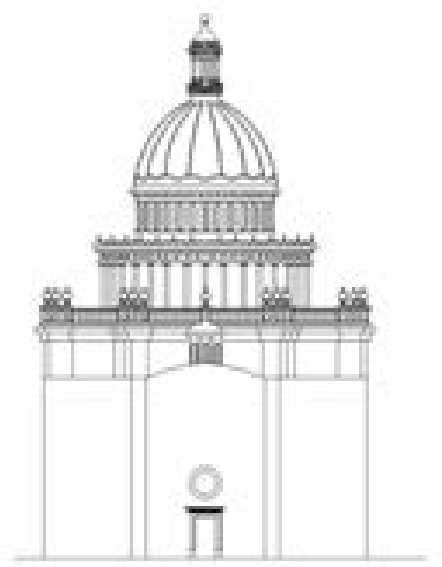

Fachada posterior

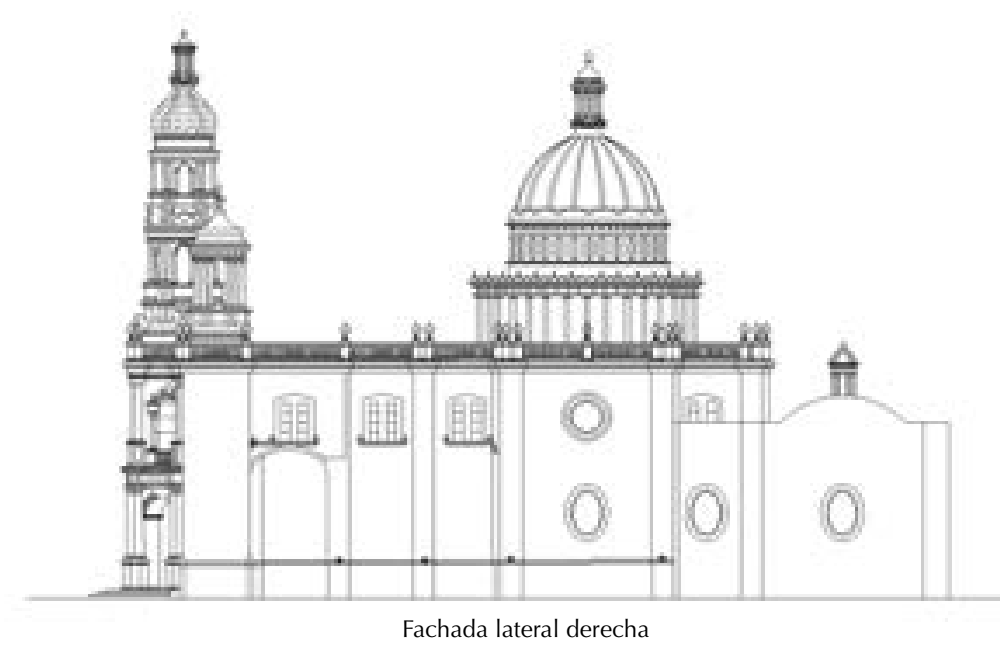

FIGURA 4. Detalles arquitectónicos del templo de San Antonio de Padua; planta arquitectónica, fachada principal, posterior y lateral (Ilustración: Edith Orenday-Tapia, 2019; fuente: M. Navarro 2013).

estilo barroco salomónico terminado en forma clásica de trapecio; el cuerpo central posee un par de columnas a cada lado de la puerta principal, las cuales enmarcan las estatuas de cantería. Se ingresa al recinto a partir de un arco de medio punto, adornado con guirnaldas y rosetones labrados. Posee, además, dos torres, una a cada lado; la primera de ellas se construyó en 1764 y la segunda en 1946 (Corpus 1969).

La nave central mide $58.40 \mathrm{~m}$ de largo y $27.30 \mathrm{~m}$ de ancho, y las naves laterales tienen un promedio de 30.00 $\mathrm{m}$ de largo y $6.80 \mathrm{~m}$ de ancho. La cúpula principal, que se ubica en la parte central del templo, está compuesta por seis lados. La capilla de Lourdes y la zona de criptas se localizan a los costados de la fachada (Padilla 2017).

A pesar de que el edificio se ha intervenido a lo largo de su vida, en 2012 comenzó a mostrar daños:

ocasionado por asentamientos generados por movimientos en el subsuelo debido a causas de la humedad y presencia de oquedades (criptas) [...] Los principales problemas estructurales generados por los asentamientos son: generación de grietas [...] las paredes exteriores muestran deterioro a causa de los efectos del intemperismo y humedad, este deterioro se hace más notorio principalmente en la nave mayor, ya que pertenece a la primera etapa de construcción de la iglesia [Padilla 2017: 35)].

Templo de San Antonio de Padua

Ubicado en el centro de la ciudad de Aguascalientes (Figura 2B), es de estilo ecléctico: neorrenacentista, neobarroco, neogótico, neoclásico, árabe, ruso, entre otros (Gobierno del Estado de Aguascalientes 2010). Fue erigido entre los 1895 y 1908, diseñado y construido por el zacatecano autodidacta Refugio Reyes (Reyes 2013).

La planta tiene forma de cruz latina (Figura 4), con un nártex en donde se localiza el coro; mide $38.81 \mathrm{~m}$ 
de largo por $21.25 \mathrm{~m}$ de ancho, dejando de lado la capilla de Santa Rita de Casia, que se encuentra en la parte posterior del templo (Saldívar 2015). El sistema constructivo es a base de muros de carga de mampostería, con arcos de medio punto y columnas (Villegas 1974). Los muros de carga son de mampostería elaborada principalmente con sillar de matacán (un limo arcilloso pobremente litificado), unido con mortero de arena y cal apagada.

"De la construcción sobresale su fachada, la cual es de sillar de cantera de tres colores (verde, amarilla y rosa)" (Navarro 2013: 22), la cual es simétrica, compuesta por dos cuerpos horizontales coronados por tres torres, una principal al centro y más alta que las demás y dos torreciIlas laterales secundarias.

La cúpula está formada a partir de un sistema estructural mixto, que combina la piedra de cantera con una serie de anillos metálicos hechos a base de rieles; compuesta por dos cuerpos, el primero formado por un tambor sin muros, sostenido por una fila triple de columnas dóricas concéntricas separadas; en el segundo cuerpo con un pretil ondulante en el eje de las columnas. Posteriormente se encuentra la cubierta peraltada y, por último, la alargada linternilla (Villegas 1974). Las cubiertas están formadas por bóvedas de crucería, de vigueta y bóveda de ladrillo (Navarro 2013), cuyas nervaduras terminan unidas por un anillo del mismo grosor que enmarca el rosetón de donde salen las lámparas (Villegas 1974).

No se sabe con certeza cuántas intervenciones ha sufrido el templo, sin embargo, se destacan algunas, como la construcción de criptas subterráneas que conectan la capilla de Santa Rita con la sacristía y la colocación de vigas metálicas en el nártex del acceso principal. Además, en 2008 se realizaron trabajos de limpieza de la cantera, inyección de grietas, reintegración de piezas faltantes de los elementos ornamentales, consolidación de piezas deterioradas y reintegración de color en la cúpula y las torres (Navarro 2013).

\section{Técnicas de intervención no destructivas}

Cuando se habla de intervenir un edificio histórico, la connotación es la recuperación respetuosa del patrimonio, que requiere especialistas en la materia para la elaboración de proyectos, las investigaciones pertinentes y los análisis necesarios, la dirección y supervisión de la obra, con el fin de lograr que las propuestas en el proyecto se apliquen de manera correcta (Terán 2004). Sin embargo, para intervenir un edificio histórico desde una perspectiva integral, protegiendo tanto los valores físicos como intrínsecos, es necesario tener un "entendimiento total" de aquél (Peña, 2008). Por lo tanto, para efectuar la intervención —una restauración, rehabilitación o consolidación- de un edificio patrimonial, es necesario primero realizar su diagnóstico y evaluación. Según Peña
(2008), tales diagnósticos y evaluaciones se dividen en cualitativos y cuantitativos.

\section{Diagnóstico cualitativo de estructuras religiosas de Aguascalientes}

Los diagnósticos cualitativos corresponden a la información que se obtiene de la estructura, la que generará datos sobre sus materiales, sistemas constructivos, historia, entre otros. Los trabajos se orientan hacia la observación del daño estructural, la degradación de los materiales y la investigación histórica de éstos.

Algunos resultados obtenidos de los diagnósticos cualitativos son la identificación de los elementos con daño de la estructura que deberán intervenirse, el análisis de información documental —o de informantes clave- sobre el sistema constructivo de un edificio, la ubicación del banco de material de donde se extrajeron las piedras con las que se levantó la construcción, entre otros. Dentro de los métodos cualitativos que se han utilizado en la UAA, se identificaron tanto la observación de patologías como el uso de la fotogrametría para la creación de modelos tridimensionales de los edificios.

\section{Observación de patologías}

La patología constructiva es la "ciencia que estudia los problemas constructivos que aparecen en el edificio o [en alguna de sus unidades] después de su ejecución" (Cedeño 2008: 73). En otras palabras, estudia el daño que existe en una estructura, ya sea que aparezca a pocos meses de su ejecución o después de varios años, pudiendo ser ocasionados por el deterioro normal del tiempo o la intemperie, por algún desastre natural, como sismos o fallas geológicas, o por una causa antropogénica.

En 2013, Navarro realizó un levantamiento de los daños del templo de San Antonio. Entre los hallazgos identificó la existencia de algunas lesiones físicas y mecánicas en distintas partes del edificio, como fisuras, humedades y piezas faltantes. Con base en la información obtenida se realizaron fichas técnicas como las que se muestran en la Figura 5, donde se registró la ubicación del daño, una fotografía y una descripción de cada uno de los elementos deteriorados.

No se sabe si las fisuras reportadas en el levantamiento se debieron a la remodelación que se hizo en las calles aledañas al templo o si, en cambio, ya existían en el edificio y se descubrieron gracias a las obras que se hicieron en la zona.

Otro estudio importante dentro de este tipo de métodos es el acopio de información histórica, de tal forma que, en la medida de lo posible, se conozcan los procesos constructivos, las etapas de construcción, la procedencia de los materiales utilizados en la construcción, 

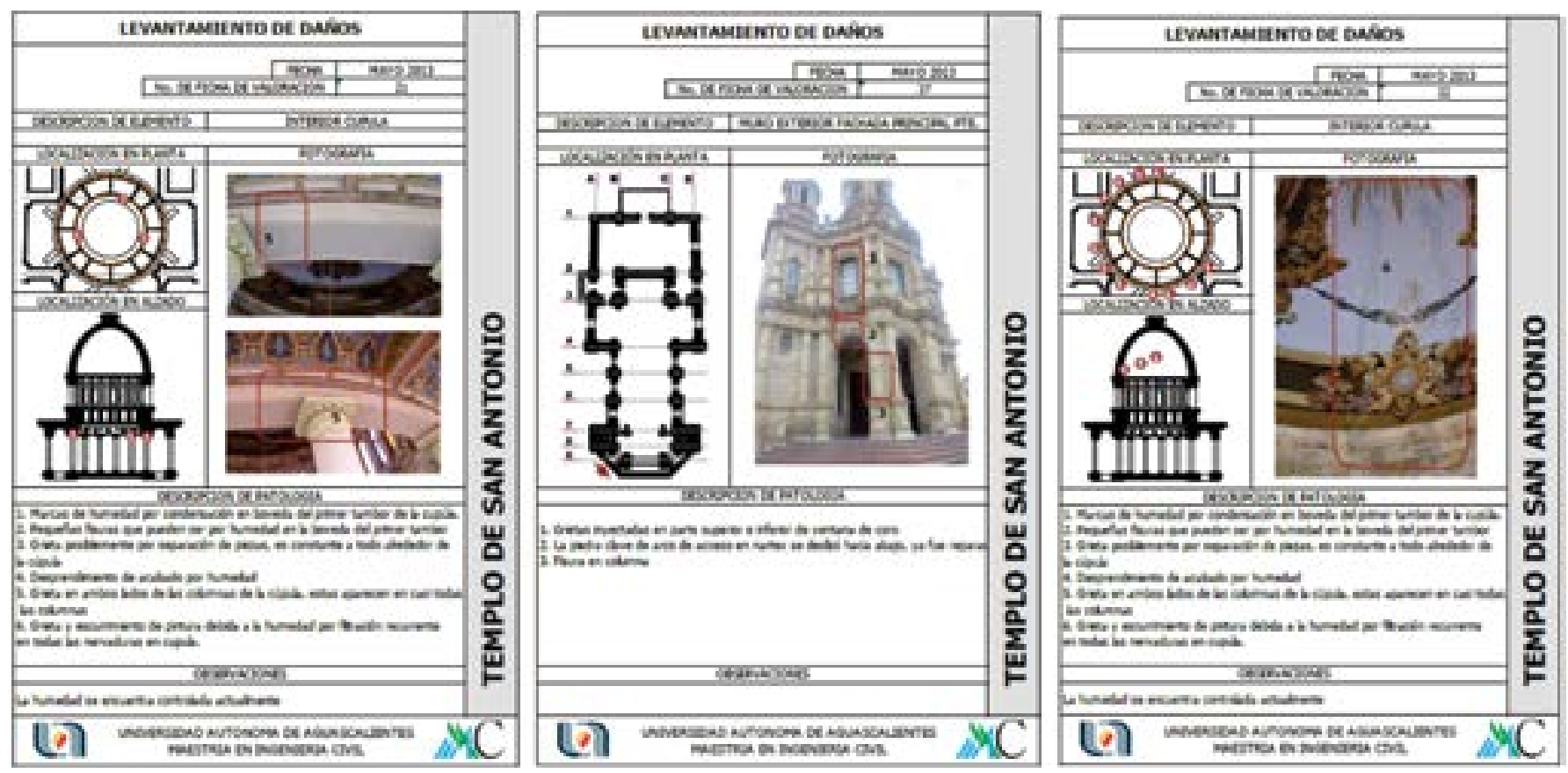

FIGURA 5. Fichas de levantamiento de daños del templo de San Antonio de Padua (Ilustración: Edith Orenday-Tapia, 2019; fuente: M. Navarro 2013).

las intervenciones realizadas en el inmueble, entre otros, ya que el patrimonio cultural está íntimamente ligado a la memoria colectiva y a los productos tangibles, que son una verificación objetiva de las huellas del pasado.

El acopio de información histórica constituyó un apartado importante en los trabajos del estudio de la Catedral de Aguascalientes (Padilla 2017) y del templo de San Antonio (Navarro 2013; Orenday 2016), presentados previamente. Éstos sirvieron de base para identificar el tipo de materiales utilizados en los edificios, sus edades, la ubicación de la que se extrajeron las canteras con las que se construyeron y, así, por un lado, dirigir los estudios que debían realizarse y, por el otro, interpretar sus resultados.

\section{La fotogrametría como herramienta \\ para el diagnóstico de un edificio patrimonial}

La fotogrametría es una técnica que utiliza la fotografía para definir con precisión la forma, las dimensiones y la posición de un objeto cualquiera. Entre las ventajas que ofrece este método incipiente están la representación digital completa de cualquier objeto y la obtención objetiva, instantánea, cómoda y económica de la información (Castro 2016); por sus ventajas, los investigadores lo han empleado para obtener modelos en 2D y en 3D de zonas arqueológicas (Bryan y Chandler 2008) y para el estudio de edificios patrimoniales en la ciudad de Aguascalientes.

"La virtualización del patrimonio edificado por medio de técnicas fotogramétricas y modelado en 3D representa una importante alternativa en el estudio del patrimonio edificado. Aporta una visión global del inmueble y muestra detalles importantes en zonas de difícil acceso" (Padilla et al. 2015: 373). Esta técnica permite obtener la disposición, las medidas y las formas de los elementos desde una perspectiva global o local: su metodología consta de la obtención de información (por fotogrametría terrestre y aérea, escáner láser terrestre y aéreo y el vehículo aéreo no tripulado, esto es, dron), el procesamiento de aquélla y de los resultados (modelo).

Padilla et al. (2015) utilizaron la fotogrametría para modelar en 3D la Catedral de Aguascalientes y determinar si, a partir de este método, se podrían identificar de manera precisa las características geométricas y físicas del edificio. Para la elaboración del modelo se tomaron las fotografías a partir del uso de drones para visualizar zonas de difícil acceso (vuelos a $40 \mathrm{~m}$ de altura, aproximadamente) y de fotografías obtenidas de forma manual, con lo que se recopilaron 1500 imágenes (Padilla 2017). Sin embargo, debido a la complejidad del edificio, el modelo no se logró completar en su totalidad (aproximadamente $80 \%$ del edificio), pero se modelaron aspectos importantes de la geometría de la construcción y datos de sus condiciones actuales. En la Figura 6 se observa el modelo del levantamiento de la Catedral de Aguascalientes mediante fotogrametría. Una vez realizado, se identificaron algunas patologías en el edificio, como agrietamientos, desprendimiento de elementos y daños ocasionados por intemperismo.

No obstante que el modelo no se completó, el estudio significa un avance importante en la aplicación de la fotogrametría a gran escala para evaluar edificios patrimoniales desde una perspectiva global y local: permite conocer características geométricas y contar con un modelo vectorizado que puede exportarse rápidamente a 
programas de análisis numérico y conseguir información sobre el estado estructural del edificio.

Diagnóstico cuantitativo de edificios religiosos en Aguascalientes

Los métodos no destructivos - también conocidos como no invasivos o non-destructive test (NDT) - se han empleado para diagnosticar edificios que, sin daño físico aparente, demandan que se conozca su condición estructural; también en aquellos en los que existen signos de deterioro, como agrietamientos, fisuras, desprendimiento de materiales, por mencionar algunos, cuya estabilidad estructural es necesario determinar.

Los NDT pueden aplicarse a los materiales o a la estructura para obtener información sobre su forma (configuración interna o externa), condición o propiedades físicas sin causar daño a ninguna de sus partes (Vekey 1988). Aunque varios de estos métodos se han desarrollado específicamente para el diagnóstico de la mampostería, algunos corresponden a otras áreas del conocimiento, como la arqueología, la aeroespacial, la geofísica u otras aplicaciones (Harvey Jr. y Schuller 2010; Schuller 2006), y se han adaptado para las condiciones que presentan las edificaciones de mampostería. Cuatro métodos se han empleado en los templos antes mencionados de la ciudad de Aguascalientes: la evaluación del deterioro de la mampostería, la caracterización de las propiedades mecánicas de la mampostería, el uso de software para el análisis estructural y la caracterización dinámica.

\section{Evaluación del deterioro de la mampostería}

Padilla et al. (2017) ejecutaron una investigación en la Catedral de Aguascalientes, donde se realizaron estudios petrológicos (difractometría de rayos $\mathrm{X}$ ) y petrofísicos (porosidad, densidad y densidad aparente) en piedras de mampostería del templo tomadas de anteriores trabajos de restauración así como el levantamiento de daños en las piedras de la mampostería y la clasificación de los tipos de roca utilizados para elaborar los muros, como lo muestra la Figura 7.

Se identificaron los diferentes tipos de piedra de los que estaba constituido el edificio para, más adelante, realizar pruebas de laboratorio in situ, como de Karsten, captación de agua, distribución de radios de poro y cristalización de sales. Con los resultados obtenidos se identificó el tipo de piedra más propenso a deteriorarse, el mecanismo de deterioro y los factores que lo aceleran.

Los resultados de Padilla et al. (2017) mostraron que la mampostería de la catedral fue elaborada usando fragmentos de rocas volcánicas ácidas (riolitas-ignimbritas), diferenciándose tres tipos de acuerdo con la zona de donde se extrajeron: toba Barranca, toba Valladolid

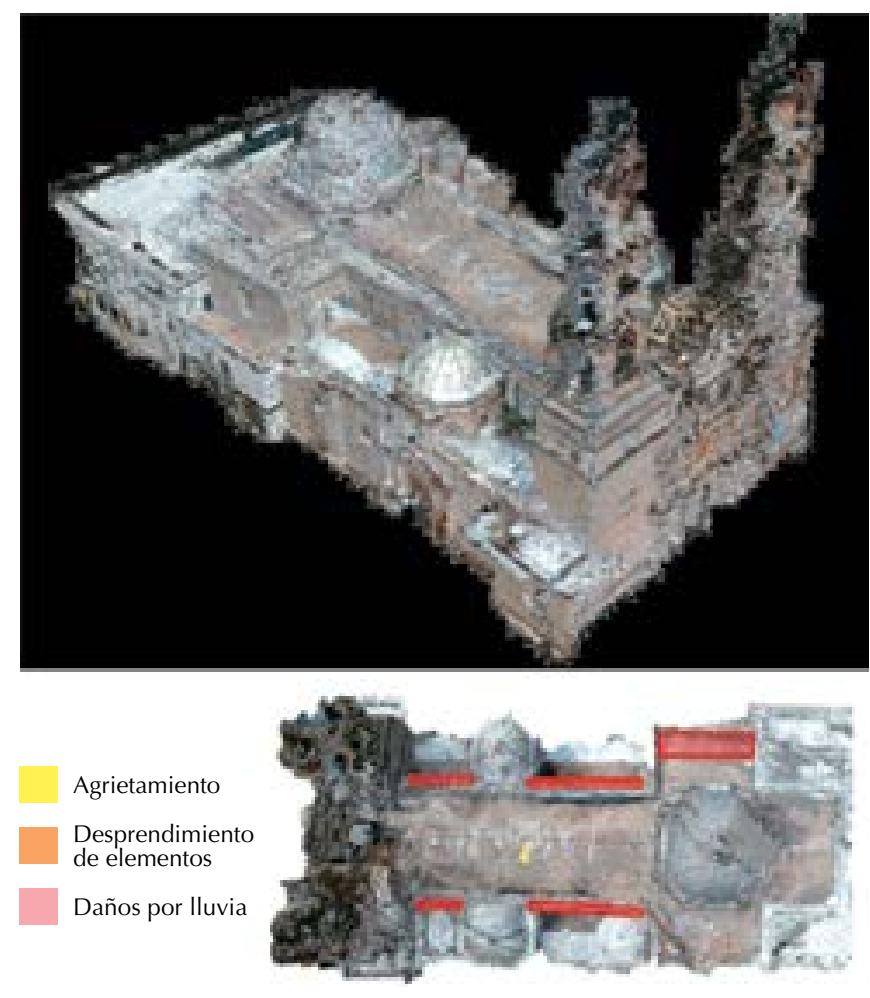

FIGURA 6. Modelo tridimensional y planta de la Catedral de Aguascalientes a partir de fotogrametría (Ilustración: Edith Orenday-Tapia, 2019; fuente: R. Padilla et al. 2015).

y toba San Miguel el Alto. La mampostería original fue construida con fragmentos de roca de la toba Barranca; la toba Valladolid se usó durante la restauración de la catedral en la década de 1970, y la toba de San Miguel el Alto se empleó para la restauración más reciente, de 2014. Padilla et al. (2017) concluyeron que la toba de la mampostería de la catedral está expuesta a un proceso de degradación por efecto de la cristalización de sales, favorecida por la humedad, principalmente, en la parte baja de los muros del edificio.

\section{Caracterización de las propiedades mecánicas} de la mampostería

Orenday (2016) realizó un estudio para la determinación de las propiedades mecánicas de la mampostería a partir de un método no destructivo que se basa en el análisis de la propagación de ondas sísmicas. En la Figura 8 se sintetiza la metodología que se usó, la cual incluye la medición de los tiempos de viaje de las ondas elásticas generadas artificialmente (a cortante: $V s$, y compresión: $V p$ ), para determinar su velocidad de propagación, utilizando el mismo principio empleado en la prospección geofísica para recabar datos acerca de la composición del subsuelo (Sheriff y Geldart 1991).

Con las velocidades de onda se utilizaron las ecuaciones de la teoría elástica para obtener las propiedades 

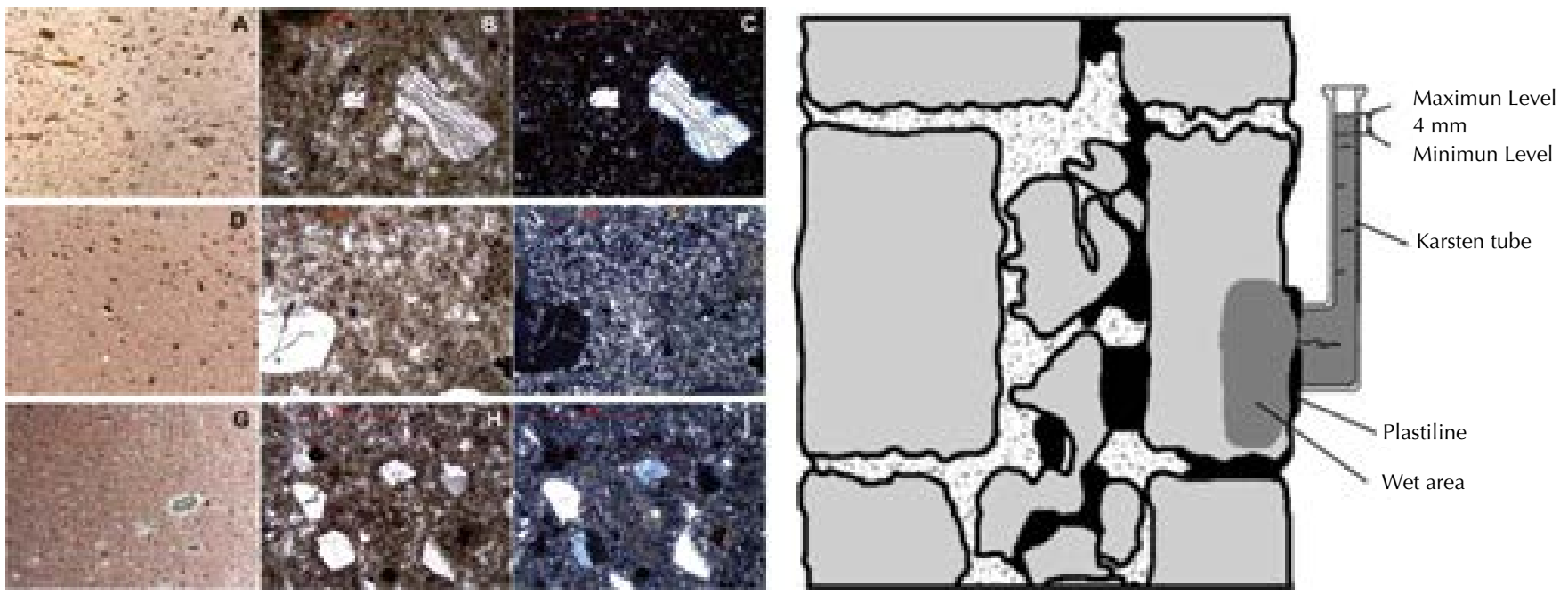

FIGURA 7. Pruebas no destructivas realizadas a piedras de la Catedral de Aguascalientes (Ilustración: Edith Orenday-Tapia, 2019; fuente: R. Padilla et al. 2017).
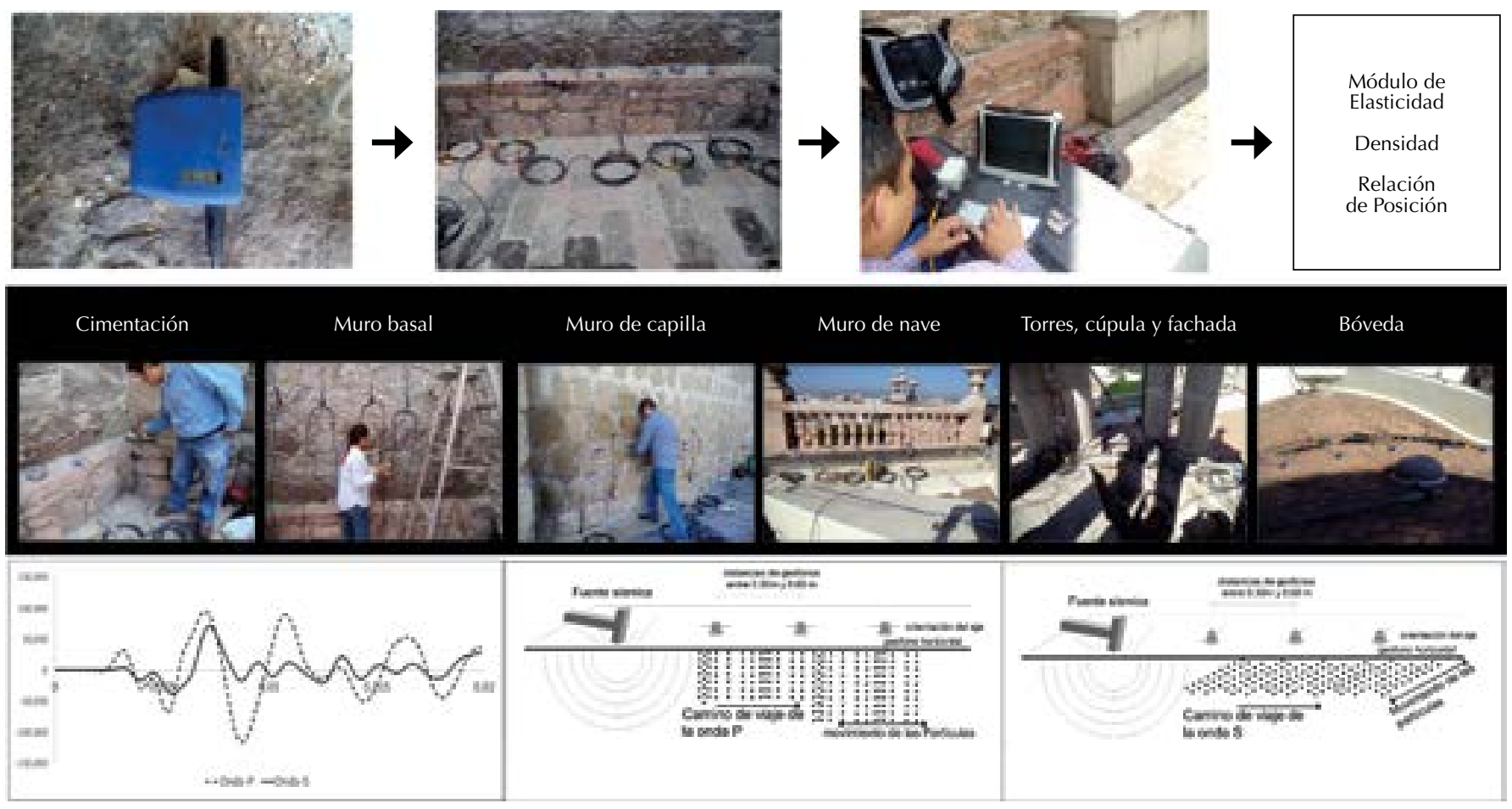

FIGURA 8. Generación y medición en campo de las ondas elásticas y su posterior análisis para la obtención de las propiedades mecánicas de los seis sistemas constructivos del templo de San Antonio de Padua (Fuente: Orenday-Tapia et al. 2019).

mecánicas de dicho material, tales como el módulo de Young, el peso volumétrico y la relación de Poisson (Orenday 2016; Orenday-Tapia et al. 2019; 2018). Estos valores son parámetros para determinar la resistencia de los materiales.

Se analizaron los seis sistemas constructivos que identificó Navarro (2013) en el templo de San Antonio de Padua (Orenday 2016; Orenday-Tapia et al. 2019; 2018), y se obtuvieron los valores de cada uno de ellos (Figura 9).

Dado que no se pueden tener muestras del templo para comparar los valores obtenidos con los valores de pruebas a compresión (método tradicional), se localizaron valores teóricos y experimentales reportados por otros autores y normas italianas para determinar si los valores obtenidos tenían coherencia respecto de los valores correspondientes con el tipo de piedra de que se trataba. En la información derivada se observó que la mayoría de los valores obtenidos en este estudio, y los valores encontrados en la literatura, no varían considerablemente; sin embargo, es necesario validar el método (para conocer los parámetros que se consideraron en este estudio (cfr. Orenday-Tapia et al. 2019; 2018). 


\begin{tabular}{|c|c|c|c|c|c|}
\hline \multirow{2}{*}{ SISTEMA CONSTRUCTIVO \DATOS } & \multicolumn{2}{|c|}{$\begin{array}{l}\text { VELOCIDAD DE ONDA } \\
\text { (PROMEDIO) }\end{array}$} & \multicolumn{3}{|c|}{$\begin{array}{l}\text { PROPIEDADES MECÁNICAS CARACTERIZADAS } \\
\text { (PROMEDIO) }\end{array}$} \\
\hline & $\mathrm{V} \rho(\mathrm{m} / \mathrm{s})$ & Vs $(\mathrm{m} / \mathrm{s})$ & $\mathrm{P}\left(\mathrm{kg} / \mathrm{m}^{3}\right)$ & $E_{d}(M p a)$ & $\square$ \\
\hline Cimentación & 2002 & 955 & 2060 & 5103 & 0.32 \\
\hline Muro basal & 1478 & 748 & 1920 & 2820 & 0.31 \\
\hline Muro de capilla & 638 & 370 & 1560 & 530 & 0.23 \\
\hline Muro de nave principal & 895 & 497 & 1690 & 1060 & 0.27 \\
\hline Muro de fachada, torres y domo & 1294 & 655 & 1860 & 2100 & 0.32 \\
\hline Bóvedas & 2313 & 1001 & 2150 & 5980 & 0.38 \\
\hline
\end{tabular}

FIGURA 9. Velocidades de onda y propiedades mecánicas caracterizadas de seis sistemas constructivos del templo de San Antonio (Tabla: Edith Orenday-Tapia, 2019).

Por tal razón, la investigación sigue en curso. Se está experimentando en especímenes de mampostería de diferentes tipos de roca y tabique, los cuales serán evaluados con el método que aquí se propone y, posteriormente, se realizarán pruebas destructivas (a compresión) para determinar el grado de variación que existe entre estos dos métodos.

Esos datos podrán consultarse cuando se realicen análisis, a partir de modelaciones numéricas, para determinar el comportamiento estructural del edificio, ya que servirán como datos de entrada de las propiedades mecánicas de los materiales de los modelos numéricos.

Software aplicado al diagnóstico estructural de edificios históricos

El análisis de edificios patrimoniales religiosos implica un alto grado de complejidad: por un lado, por la geometría de los edificios, y por el otro, por el material con el que están construidos. Afortunadamente, gracias al avance del desarrollo de software de análisis numérico, se pueden practicar análisis complejos para el estudio de las construcciones y conocer el comportamiento de las estructuras.

El método de elemento finito (FEM, por sus siglas en inglés) permite calcular esfuerzos y desplazamientos, y realizar diferentes tipos de análisis (lineal, no lineal, estático, dinámico, etc.). La selección del análisis por realizar dependerá de la información con que se cuente y los resultados que se deseen obtener así como de la disponibilidad y compatibilidad del equipo y el software (Peña 2008).

El análisis estructural de edificios patrimoniales religiosos de Aguascalientes se ha trabajado por Navarro (2013), Ánimas et al. (2013), Orenday (2016) y Hernández (2018), quienes han realizado modelos del templo de San Antonio para su análisis estructural y determinar, así, su estado actual, como se observa en la Figura 10. El propósito final de un análisis numérico es conocer las zonas críticas de la estructura donde los esfuerzos (de tensión o compresión) son muy grandes y pueden poner en riesgo la estabilidad del edificio.

Los resultados muestran que, en el templo de San Antonio, el efecto con mayor potencial de daño es el causado por la vibración del tránsito vehicular (Hernández 2018), y que los elementos más vulnerables a experimentar algún tipo de daño por dichas vibraciones son las dos torres de sus campanarios y la cúpula principal (Ánimas et al. 2013; Orenday 2016). Las indagaciones hechas por Navarro (2013) reportan una fisura vertical en el muro de la fachada principal del templo, observada por primera vez después de que las calles aledañas al templo se abrieron a la circulación de camiones del sistema de transporte público en la década de 1980.

\section{Caracterización dinámica de edificios patrimoniales}

La caracterización dinámica de un edificio consiste en medir las vibraciones de la estructura por efecto del ruido ambiental para determinar su frecuencia natural y modos de vibrar. Los datos obtenidos permiten validar los resultados de los análisis numéricos que se hacen del edificio, comparando los parámetros dinámicos determinados experimentalmente con los obtenidos del análisis modal del modelo numérico. Se usan, además, para identificar condiciones de daño estructural y patrones de degradación o pérdida de propiedades de resistencia de los materiales (Ánimas 2015). La finalidad principal del estudio de Ánimas (2015) fue diseñar una metodología para evaluar la salud estructural de monumentos y edificios históricos, utilizando el templo de San Antonio de Padua como caso de estudio.

Los resultados de Ánimas (2015) fueron corroborados por Hernández (2018) y evidencian que el templo de San Antonio tiene una frecuencia natural de vibrar de $3.8 \mathrm{~Hz}$. Adicionalmente, Hernández (2018) comprobó que las ráfagas del viento que inciden sobre el edificio y el repicar de las campanas producen vibraciones sobre éste, 

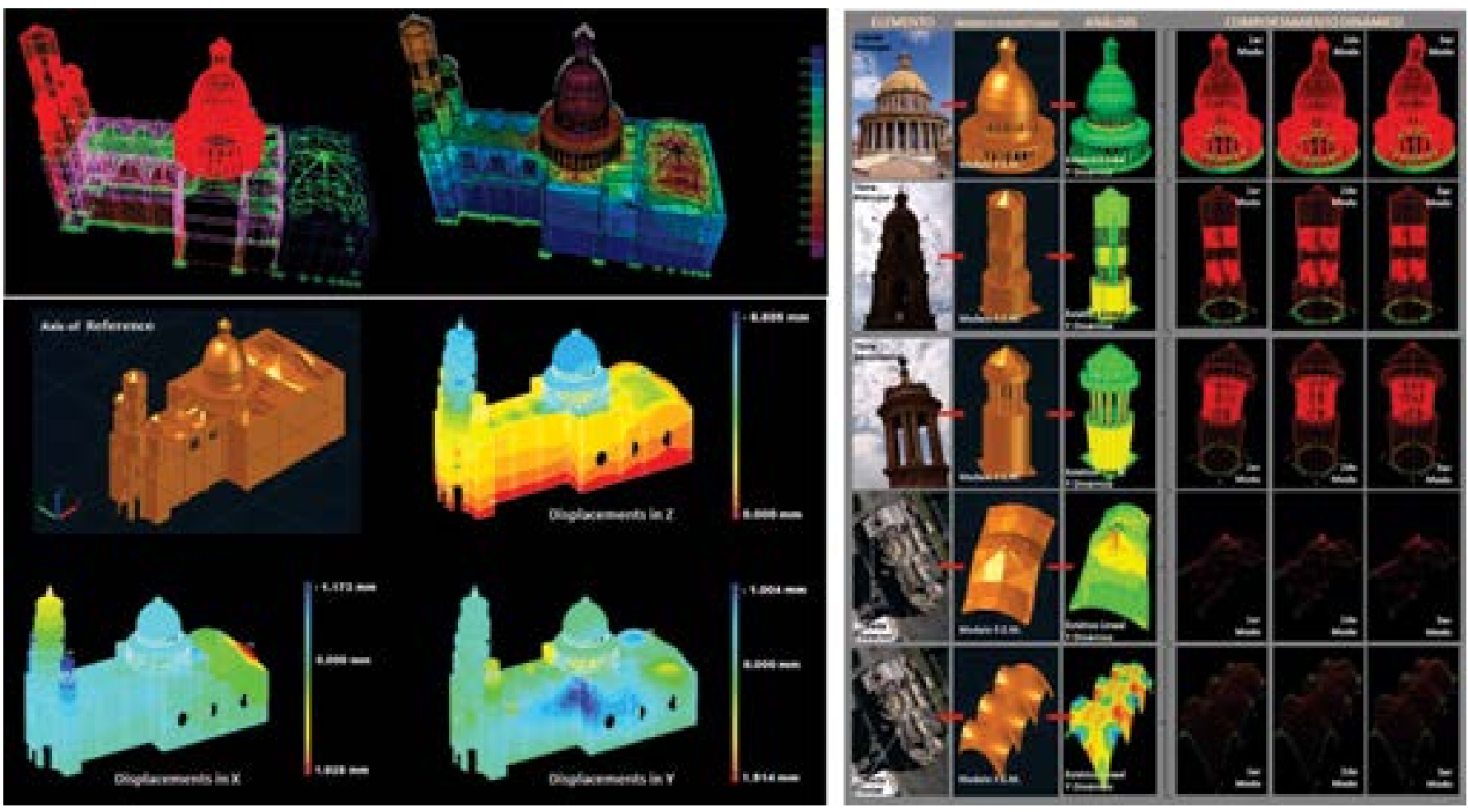

FIGURA 10. Análisis estructural a partir de modelos numéricos (Ilustración: Edith Orenday-Tapia, 2019; fuentes: H. Ánimas et al. 2013, Ánimas et al. 2014, Orenday 2016)

aunque son de menor intensidad que las causadas por el tránsito vehicular.

\section{Consideraciones finales y perspectivas}

Aunque en Aguascalientes el número de personas que profesan el catolicismo, identificadas como los "usuarios" principales de los edificios religiosos, muestra una tendencia a disminuir, la conservación de dichas construcciones es una prioridad para las autoridades locales debido a que conforman parte del patrimonio histórico, cultural y arquitectónico de la ciudad.

En Aguascalientes, el estudio de estos edificios hasta hace poco se limitaba a aspectos históricos, arquitectónicos y estéticos; no obstante, en ocasiones especiales, cuando mostraban deterioros visibles, también se hacían estudios de levantamiento de daños para orientar los trabajos de restauración. Sin embargo, los estudios de comportamiento estructural de dichos edificios se realizaron a partir de 2015, cuando el grupo de IEEP de la UAA comenzó a analizarlos desde este punto de vista.

Los estudios de la Catedral de Aguascalientes y el templo de San Antonio de Padua han proporcionado información sobre su estado actual y sus materiales así como acerca de los sistemas constructivos empleados y la estabilidad de la estructura en general. Adicionalmente, con la caracterización dinámica de los edificios mediante registros de vibración ambiental, se han calibrado y validado los modelos numéricos elaborados para simular su comportamiento estructural, logrando un incremento en la confiabilidad de los resultados de la simulación numérica. Por otra parte, se han iniciado estudios del proceso de degradación de los materiales debido al intemperismo, para entender de qué manera cambia la rigidez global del edifico y su comportamiento estructural.

Todos los estudios sobre estos dos edificios religiosos muestran que, a pesar de que han sido afectados por diversos factores (intemperismo, tránsito vehicular, degradación de los materiales, etc.), no están en peligro de colapso; sin embargo, debido a su importancia y a que continúan recibiendo a los feligreses, se hace necesario conservarlos en monitoreo y prever trabajos de mantenimiento para asegurar su preservación.

Aunque previamente se han realizado intervenciones en estos y otros edificios patrimoniales de la ciudad, las investigaciones realizadas en los dos templos aquí referidos aportan nuevos elementos para formular propuestas de intervención integral, previendo aspectos tanto estético-arquitectónicos como desde el punto de vista de la estabilidad estructural.

Actualmente existe interés del Centro INAH Aguascalientes por los resultados de los estudios del comportamiento estructural de los edificios obtenidos por el grupo de IEEP. Se espera que en el futuro cercano esos estudios se integren a los diagnósticos que se realizarán en las propuestas de consolidación y restauración de las 
construcciones históricas, para que, con estos estudios inter y transdisciplinarios, se pueda preservar tanto la belleza arquitectónica de los edificios patrimoniales como el valor religioso que sostiene la identidad cultural de la población de la localidad.

\section{Agradecimientos}

Agradecemos a la Universidad Autónoma de Aguascalientes (UAA, México), al Consejo Nacional de Ciencia y Tecnología (Conacyt, México), a la parroquia del templo de San Antonio de Padua y a la Catedral de Aguascalientes, por su apoyo durante los trabajos de investigación.

\section{Referencias}

Aguilera, P.

2013 Catedral Metropolitana. Hundimiento y rescate. México, Instituto de Ingeniería, Universidad Nacional Autónoma de México.

Ánimas, $\mathrm{H}$.

2015 "Evaluación del empleo del modo de vibrar como indicador de la condición estructural de edificios históricos", tesis de doctorado en ciencias de los ámbitos antrópicos. México, Universidad Autónoma de Aguascalientes.

Ánimas, H., J. Pacheco y J. Ortiz

2014 "Evaluation of the structural health of heritage buildings by the use of vibration-based damage detection methods", en Cultural HELP 2014. Cultural Heritage and Loss Prevention, at Porto, Portugal, pp. 95-106, documento electrónico disponible en [https://www.researchgate.net/publication/269701954_Evaluation_of_the_structural_health_of_heritage_buildings_by_the_use_of_vibration-based_damage_ detection_methods], consultado en mayo de 2018.

Ánimas, H., M. Navarro, J. Pacheco, J. García, M. Arroyo, T. Cordero, y C. Esparza.

2013 "Morfología y comportamiento estructural del templo de San Antonio", en 3er Foro Internacional en Ciencias de los Ámbitos Antrópicos y Jornadas Internacionales de Investigación, Aguascalientes, México, Universidad Autónoma de Aguascalientes/Universidad de Alcalá de Henares.

Bryan, P., y H. Chandler

2008 "Cost-effective rock-art recording within a non-specialist Environment", The International Archives of the Photogrammetry, Remote Sensing and Spatial Information Sciences 36: 259-264.

Castro, $M$.

2016 “Evaluación y calibración de las técnicas de fotogrametría digital aplicadas a la caracterización geométrica de obras patrimoniales", tesis de maestría en ingeniería civil, México, Universidad Autónoma de Aguascalientes.

Cedeño, A.

2008 "Valoración de las condiciones de la estructura antigua", Diseño y Sociedad 25-26: 72-81.
Corpus, R.

1969 Aguascalientes. La Catedral y el cabildo. Aguascalientes, Imprimatur.

De Solà-Morales, I.

2015 [1982] "Teorías de la intervención arquitectónica", Cuatro Cuadernos, documento electrónico disponible en [https://cuatrocuadernos.wordpress.com/teorias-de-la-intervencion-arquitectonica/], consultado en diciembre de 2018.

Gobierno del Estado de Aguascalientes

2010 Templo de San Antonio [Turismo], documento electrónico disponible en [http://www.vivaaguascalientes.com/UI/ frm_Datos.aspx?IA=373], consultado en febrero de 2015.

Gutiérrez, J. A.

2003 El siervo de Dios monseñor José de Jesús López y González, México, Universidad Autónoma de Aguascalientes/Obispado de Aguascalientes/Congregación Maestras Católicas.

2006 Historia de la Iglesia católica en Aguascalientes (3 vols.), México, Universidad Autónoma de Aguascalientes/Obispado de Aguascalientes.

2007a Cien años de vida de la Diócesis de Aguascalientes, México, Universidad Autónoma de Aguascalientes.

2007b La labor social de la Iglesia católica en Aguascalientes, México, Universidad Autónoma de Aguascalientes.

Harvey Jr. D., M. Schuller

2010 "Nondestructive evaluation: structural performance of masonry", Practice Points 9: 1-8, documento electrónico disponible en [http://www.apti.org/clientuploads/publications/ PracticePoints/PracticePoints9.pdf], consultado en mayo de 2018.

Hernández, L.

2018 "Medición y caracterización de vibraciones en tiempo real de estructuras para el estudio de su comportamiento", tesis de maestría en ingeniería civil, México, Universidad Autónoma de Aguascalientes.

Imdosoc

2015 Creer en México. Encuesta Nacional de Cultura y Práctica Religiosa, Instituto Mexicano de Doctrina Social Cristiana, documento electrónico disponible en [http://www.imdosoc. org/web/creer-en-mexico-encuesta-nacional-de-cultura-ypractica-religiosa], consultado en mayo de 2018. INAH

2018 ¿Quiénes somos?, documento electrónico disponible en [https://www.inah.gob.mx/quienes-somos], consultado en diciembre de 2018. Inegi

2010 Censo General de Población y Vivienda 2010, México, Instituto Nacional de Estadística y Geografía.

Martínez, A.

2016 "Identidades y patrimonios. Repensando la dinámica social", en Identidades y patrimonios. Encrucijadas entre lo material y lo intangible, México, Fontamara/Universidad de Guanajuato, pp. 11-27.

Muñoz, M.

1996 Fiestas populares en la región de Aguascalientes, México, Universidad Autónoma de Aguascalientes. 
Navarro, M.

2013 "Levantamiento geométrico y de daños del templo de San Antonio y análisis estructural de la situación actual", tesis de maestría en ingeniería civil, México, Universidad Autónoma de Aguascalientes.

Orenday, E.

2016 "Estudio de caracterización de las propiedades mecánicas del Templo de San Antonio para su análisis dinámico", tesis de maestría en ingeniería civil. México, Universidad Autónoma de Aguascalientes.

Orenday-Tapia, E. E., J. Pacheco-Martínez, R. Padilla-Ceniceros, H. Ánimas-Rivera, J.A. Ortiz-Lozano y D. Gaxiola-Apodaca 2019 "Characterization of the mechanical properties of ancient masonry from a non-destructive method in situ", en R. Aguilar et al. (eds.). Structural Analysis of Historical Constructions, Rilem Bookseries, pp. 370-378, DOI: 10.1007/978-3319-99441-3_39.

Orenday-Tapia, E. E., J. Pacheco-Martínez, R. Padilla-Ceniceros y R. A. López-Doncel

2018 "In situ and nondestructive characterization of mechanical properties of heritage stone masonry", Environmental Earth Sciences 77 (7): 286, DOI:10.1007/s12665-018-7473-8

Padilla, M. R.

2009 "Perfiles socioculturales de ciudadanía. Identidades urbanas y geografías mediáticas. Estudio de cinco escenarios en la ciudad de Aguascalientes", tesis de doctorado en estudios científicos-sociales en la comunicación, México, Instituto Tecnológico y de Estudios Superiores de Occidente/Universidad Jesuita de Guadalajara.

2013 "Dios en los medios. El caso de la Iglesia de Jesucristo de los Santos de los Últimos Días en Aguascalientes", en María Eugenia Patiño (coord.), Creencias y prácticas religiosas en Aguascalientes: ensayos interpretativos, México, Universidad Autónoma de Aguascalientes, pp. 103-125.

Padilla, R.

2017 "Desarrollo y adaptación de técnicas de caracterización física de sistemas constructivos en edificios patrimoniales con métodos no destructivos para la conservación del patrimonio histórico", tesis doctoral en ciencias de los ámbitos antrópicos, Aguascalientes, Universidad Autónoma de Aguascalientes.

Padilla, R., J. Pacheco, R. López y E. Orenday.

2017 "Rock deterioration in the masonry walls of the Cathedral Basilica of Aguascalientes, Mexico", Revista Mexicana de Ciencias Geológicas 34 (2): 138-149.

Padilla, R., T. Sonnemann y J. Pacheco.

2015 "Reconocimiento de daños y patologías de zonas de difícil acceso de la catedral basílica de Aguascalientes mediante el UAVPhantom II Vision Quadcopter", en Convergencias del diseño y de la construcción IV. Arquitectura, ingeniería civil y urbanismo. Sociedad, patrimonio urbano y sustentabilidad, México, Universidad Autónoma de Aguascalientes, pp. 370-386.

Padilla, Y.

2013 "Creencias y tolerancia religiosa en Aguascalientes: una aproximación histórica y social", en M. E. Patiño (coord.),
Creencias y prácticas religiosas en Aguascalientes: ensayos interpretativos, México, Universidad Autónoma de Aguascalientes, pp. 77-101.

Patiño, M. E.

2013 "Las creencias y las prácticas religiosas a través de las encuestas en México; un acercamiento desde las cifras", en M. E. Patiño (coord.). Creencias y prácticas religiosas en Aguascalientes: ensayos interpretativos, México, Universidad Autónoma de Aguascalientes, pp. 19-48.

Patiño, M. E., y E. Tapia

2009 "Construyendo espacios: la contribución de las religiosas católicas", en S. Bénard y O. Sánchez (coords.), Vivir juntos en una ciudad en transición. Aguascalientes frente a la diversidad social, México, Universidad Autónoma de Aguascalientes, pp. 217-241.

Patiño, M. E., y G. Zalpa

2014 "Cultura religiosa en Aguascalientes", en R. de la Torre, C. Gutiérrez, M. E. Patiño, Y. Silva, H. J. Suárez y G. Zalpa (coords.), Creer y practicar en México: comparación de tres encuestas sobre religiosidad, México, Universidad Autónoma de Aguascalientes/Centro de Investigaciones y Estudios Superiores en Antropología Social/Colegio de Jalisco, pp. 63-100.

Peña, $F$.

2008 "Estrategias para el modelado y el análisis de edificios históricos de mampostería", en Memorias del XVI Congreso Nacional de Ingeniería Estructural, Veracruz, Sociedad Mexicana de Ingeniería Estructural, pp. 1-14.

Reyes, A.

2013 Refugio Reyes, una vida. El aprendizaje, Aguascalientes, Consejo Nacional para la Cultura y las Artes. SAHC

2019 Structural Analysis of Historical Constructions, R. Aguilar, D. Torrealva, S. Moreira, M. Pando, L. F. Ramos, (eds.), Nueva York, Springer International Publishing (Rilem Bookseries, 18), documento electrónico disponible en [https://www. springer.com/us/book/9783319994406], consultado en diciembre de 2018.

Saldívar, J. A.

2015 "Historia de la orden religiosa de San Agustín en Aguascalientes. Alcances históricos, institucionales y educativos", tesis doctoral en ciencias sociales y humanidades, Aguascalientes, Universidad Autónoma de Aguascalientes.

Schuller, $M$.

2006 "Nondestructive testing and damage assessment of masonry structures", en In-situ Evaluation of Historic Wood and Masonry Structures, Praga, NSF/Rilem Workshop, documento electrónico disponible en [https://www.rilem.net/images/publis/pro062-007.pdf], consultado en mayo de 2018.

Sheriff, R., y L. Geldart

1991 Exploración sismológica, vol. I. Historia, teoría y obtención de datos, Mexico, Limusa.

Tapia, E.

2011 "Virgen de media noche y otras más... (Las mujeres en el bolero)", en Antonio Higuera (coord.), Religión y culturas contemporáneas, México, Universidad Autónoma de Aguas- 
calientes, Cuerpo Académico de Estudios de la Cultura Contemporánea-Red de Investigadores del Fenómeno Religioso en México, pp. 355-374.

Terán, J.

2004 "Consideraciones que deben tenerse en cuenta para la restauración arquitectónica", Conserva 8: 101-122, documento electrónico disponible en [http://www.cncr.cl/611/ w3-article-4673.html], consultado en mayo de 2018.

Vekey, R.

1988 "Non-destructive test methods for masonry structures", IBMAC 1988: 8th International Brick and Block Masonry Conference, 3: 1673-1681, documento electrónico disponible en [http://www.hms.civil.uminho.pt/ibmac/1988/1673.pdf], consultado en mayo de 2018.

\section{Síntesis curricular de/los autor/es}

\section{Edith Estefanía Orenday Tapia}

Doctorado en Ciencias de los Ámbitos Antrópicos,

Universidad Autónoma de Aguascalientes (UAA), México eeorenday@gmail.com

Licenciada en arquitectura (Universidad Autónoma de Aguascalientes [UAA], México), maestra en ingeniería civil con salida de especialización en estructuras (UAA) y estudiante del doctorado en ciencias de los ámbitos antrópicos (UAA). Miembro de Investigación en Ingeniería Estructural de Edificios Patrimoniales (IEEP-UAA), donde también es docente. Actualmente, investiga métodos no destructivos para la obtención de propiedades mecánicas de mampostería en edificios patrimoniales. Sus publicaciones más recientes son "In situ and Nondestructive Characterization of Mechanical Properties of Heritage Stone Masonry", en Environmental Earth Sciences (2018), y "Characterization of the Mechanical Properties of Ancient Masonry from a Non-Destructive Method in situ", en Rilem Bookseries, vol. 18 del SAHC (2019).

\section{Evangelina Tapia Tovar}

Departamento de Sociología y Antropología,

Universidad Autónoma de Aguascalientes (UAA), México

etapia@correo.uaa.mx

Licenciada en sociología (Universidad Autónoma de Aguascalientes [UAA], México), maestra en investigación en ciencias del hombre (UAA), y doctora en ciencias sociales, en la línea de estudios culturales (UAA). Profesora-investigadora de tiempo completo del Departamento de Sociología y Antropología
Villegas, V. M.

1974 Arquitectura de Refugio Reyes, México, Imprenta Madero. Zalpa, G.

2003 Las iglesias en Aguascalientes. Panorama de la diversidad religiosa en el estado, México, Centro de Investigaciones y Estudios Multidisciplinarios de Aguascalientes/Universidad Autónoma de Aguascalientes/Colegio de Michoacán.

2013 "Del dicho al hecho... ser creyentes en Aguascalientes", en M. E. Patiño (coord.), Creencias y prácticas religiosas en Aguascalientes: ensayos interpretativos, México, Universidad Autónoma de Aguascalientes, pp. 49-75.

2014 Enciclopedia de las religiones en México, México, Universidad Autónoma de Aguascalientes.

desde 1986, donde imparte cátedra y dirige tesis de licenciatura, maestría y doctorado. Miembro del Sistema Nacional de Investigadores (SNI), nivel 1, del Cuerpo Académico Consolidado Estudios Socioculturales (Prodep) y del Programa Nacional de Investigación en Rendición de Cuentas y Combate a la Corrupción (CIDE). Ha realizado investigaciones sobre religión, música y corrupción, y publicado artículos, capítulos y libros; el más reciente es "La vuelta al mundo de la corrupción en veinte refranes".

\section{Jesús Pacheco Martínez}

Departamento de Construcción y Estructuras,

Universidad Autónoma de Aguascalientes (UAA), México

jesus.pacheco@edu.uaa.mx

Ingeniero civil (Universidad Autónoma de Querétaro [UAQ], México), maestro en ciencias con especialidad en ingeniería estructural (UAQ) y doctor en ciencias de la tierra con especialización en exploración geofísica (Universidad Nacional Autónoma de México [UNAM]). Miembro del Sistema Nacional de Investigadores (SNI), nivel 1. Sus líneas de investigación son ingeniería estructural de edificios patrimoniales y ciencias de la tierra aplicada a ingeniería civil; es miembro de la Sociedad Mexicana de Ingeniería Estructural, American Geophysical Union, entre otras, y profesor-investigador del Departamento de Construcción y Estructuras de la Universidad Autónoma de Aguascalientes (UAA) desde 2006, donde imparte cátedra y dirige trabajos de tesis de licenciatura, maestría y doctorado. Ha publicado artículos indizados en JCR, libros y capítulos. 